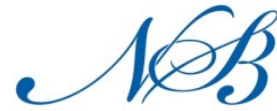

Notulae Scientia Biologicae

\title{
Ethnobotanical Knowledge Studied in Pocharam Wildlife Sanctuary, Telangana, India
}

\author{
Pendem SAIDULU ${ }^{1}$, Sateesh SUTHARI ${ }^{2}$, Ramesh KANDAGATLA ${ }^{2}$, \\ Ragan AJMEERA², Raju S. VATSAVAYA²* \\ ${ }^{1}$ Telangana State Social Welfare Residential School/Junior College, Bhongir, Nalgonda District, Telangana, 508 116, India; pendemsaidulu8@gmail.com \\ 2Plant Systematics Laboratory, Department of Botany, Kakatiya University, Warangal, Telangana, 506009, India; suthari.botany@gmail.com;rkbotany@gmail.com; \\ raganajmeera@gmail.com;rajuvatsavaya@gmail.com(*correspondingauthor)
}

\begin{abstract}
A survey was conducted in 31 fringe villages of Pocharam wildlife sanctuary, Telangana, India, during 2010 to 2012, in order to explore and document the ethnobotanical knowledge of Yerukulas and Lambadis communities. There was revealed the use of 173 Angiosperm species. The pattern of the plant use as per habitat (terrestrial/aquatic), habit (growth form), plant part (organ) and taxonomic category (families), nativity and occurrence (wild/cultivated) were established. Dicots contribute more than Monocots to the medicinal and ethnobotanical use. This might be due to the species strength in the region. When the plant use-data were analyzed, trees contributed with 68 uses, followed by herbs (51), climbers (32) and shrubs (22). Perhaps this was a reflection of the floristic composition and the prevailing Phanero-therophytic climate. Out of the 173 plant taxa that were noted as being utilized by the ethnic people in the sanctuary, the greatest number $(154 ; 89.1 \%)$ were indigenous and wild. The introduced species were the crops under cultivation and planted. Although the local people use plants for various purposes, they largely serve medicinal scopes (83.24\%) and for subsistence (21.96\%).
\end{abstract}

Keywords: ethnic use, native species, plant resource, sanctuary, wildlife

\section{Introduction}

Eighty percent of the Indian population lives in villages and a considerable proportion of it comprises the tribal communities residing in remote forest areas. The traditions, beliefs, taboos and needs of the tribes vary with the floral diversity of the habitat that dearly contributes to plant-folklore. Obviously, the plants play a vital role in the maintenance of health besides providing food, fibre and shelter which are very basic to these people.

For Telangana region in southern India, Khan (1953) was perhaps the first to record the ethnobotanical uses of some plant species. Kapoor and Kapoor (1980), Hemadri (1990) and Naqvi (2001) enumerated the medicinal plant wealth of Karimnagar district. Ramarao (1988) visited Rangapur and Thupakulagudem hamlets and interacted with Koyas and Lambadis of Warangal district for his doctoral thesis on ethnobotany of Eastern Ghats, while Ravishankar (1990) studied the ethnobotany of Adilabad and Karimnagar districts. Pullaiah and Kumar (1996) and Kumar and Pullaiah (1998) enumerated the medicinal herbs in Mahabubnagar district. Reddy et al. (1998) documented the ethnoveterinary practices in Warangal district; they made 49 additions to the Dictionary of Ethnoveterinary Plants of India (cf. Appendix by Jain SK, 1999). Certain research articles were published on the traditional botanical knowledge of Gonds of Northern Telangana (Adilabad district: Ravishankar and Henry, 1992; Swamy, 2009; Omkar et al., 2011; Karimnagar district: Reddy et al., 2002).
The ethnobotany of Khammam district received fair attention. Upadhyay and Chauhan (2000) published a brief account of ethnobotany of Gundala mandal. Reddy et al. (2001) reported the ethnic medicinal uses of the endemic Heterostemma deccanense at Sukkumamidi. Reddy (2002) documented the ethnobotanical information for 550 plants from Warangal district. Reddy and Raju (2002) published the ethnobotanical observations on Konda Reddis of Mothugudem and Raju and Reddy (2005) reported the ethnomedicine for dysentery and diarrhoea. Reddy $e t$ al. (2007) published an account of the phytotherapy practiced by Gonds in Warangal district, while Murthy et al. (2007) made a brief report of the ethnoveterinary practices in vogue among the Koyas in Pakhal wildlife sanctuary. Recently, there were investigated the medicinal and economic uses of 204 climbing plants of 132 genera, 50 families of Magnoliophyta and two ferns of northern Telangana (Suthari et al., 2014a) and the varying traditional botanical knowledge of plant medicines of Koya community inhabiting in 16 villages of Eturnagaram wildlife sanctuary area (Suthari et al., 2014b).

For Southern Telangana, Padmarao et al. (1999) published a note on folk treatment of bone fractures in Ranga Reddy district and Reddy and Raju (2000) enlisted the folklore biomedicine for common veterinary diseases in Nalgonda district. Reddy et al. (2010) reported 82 medicinal plant species of ethnic use in Medak district without mentioning any tribe. Later, Sreeramulu et al. (2013) reviewed the ethno-botanical-medicinal aspects for common human ailments in Nalgonda and Warangal districts. 


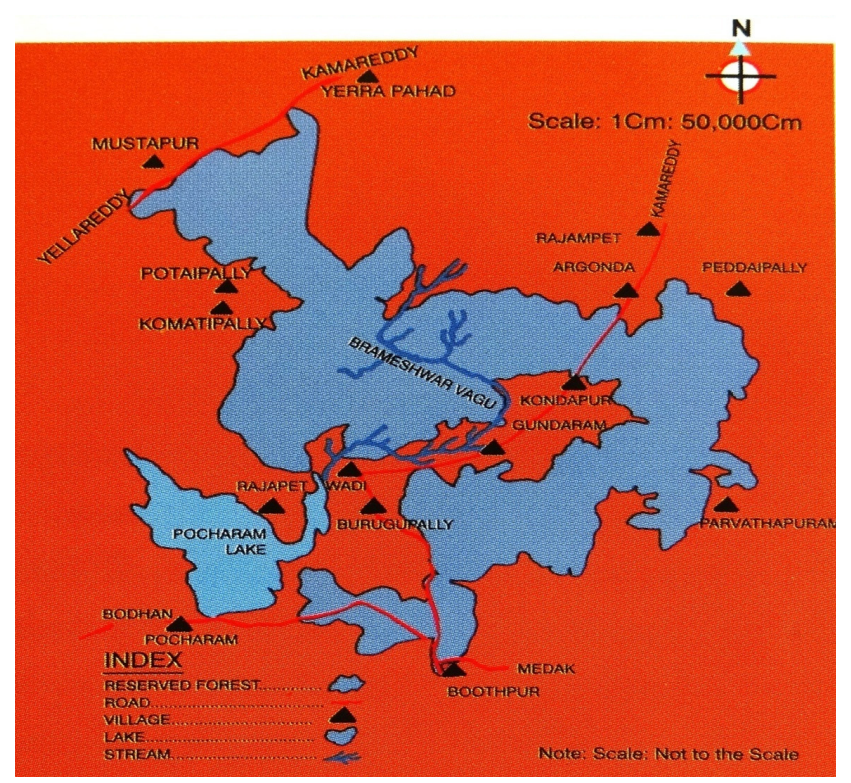

Fig. 1. The study area- outline of Pocharam wildlife sanctuary (Source: Brochure published by Andhra Pradesh Forest Department, Hyderabad)

Conversely, there is no field-level baseline of ethnobotanical data published on Pocharam wildlife sanctuary. Therefore, an attempt was made to provide an inventory of the area.

\section{Study area}

The Pocharam Lake was built during 1916-1922 near Pocharam village by damming Allair, a tributary of Manjeera River. The Pocharam wildlife sanctuary (Fig. 1) was established in 1952 around this water body and lies in between the latitudes $18^{\circ} 6^{\prime}$ to $18^{\circ} 18^{\prime} \mathrm{N}$ and longitudes $78^{\circ} 8^{\prime}$ to $78^{\circ} 20^{\prime} \mathrm{E}$. The sanctuary has two Reserve Forest blocks (dry deciduous type), namely Boothpur (1076.89 ha) and Lingampet (11907.67 ha). Presently, the wildlife sanctuary has a land area of $129.85 \mathrm{sq} \mathrm{km}$ spread in the districts of Medak (incl. Ramayampet) and Nizamabad (incl. Lingampet, Tadvai and Nizamabad).

A major portion of the sanctuary is of peninsular granite complex, basically of Archean formation. The soil varies from red to sandy loam. Heavy biotic pressure, indiscriminate felling/cutting of trees, uncontrolled grazing and annual forest fires are contributing to soil erosion and further depletion of soil nutrients. The soil has good forest growth only in valleys. In plains, the soil is compacted due to trampling. Very little percolation of water and run off occurs through this land soil surface (Anonymous, 2000). The area receives Southwest monsoon rains (June to September), with sporadic showers during Northeast monsoon season. The annual average rainfall in the sanctuary is $700 \mathrm{~mm}$. During summer, the temperature rises up to $47^{\circ} \mathrm{C}$ while it falls down to $10^{\circ} \mathrm{C}$ in winter.

\section{Tribes}

The people inhabiting the study area are largely the Yerukalas and the Lambadis. Although both are of Scheduled-tribe category, the latter are non-endemic. Yerukalas are chiefly engaged in rearing pigs, making baskets and brooms. They live in small huts built with bamboo poles and grass roofs. Lambadis are economically better developed and politically alert in comparison to the local indigenous ethnic people such as Gonds, Koyas and others in Northern Telangana. Even though the sanctuary is proximate to Adilabad (Central India - the Gond Region), neither the Gonds nor the familiar Teak forests abound. There are 53 villages within the $5 \mathrm{~km}$ border of the sanctuary, with sparsely populated ethnic people. These villagers mainly depend on forest for timber, agricultural implements, fibre, fuel, fodder and other non-timber forest produce (NTFPs). Of the abiotic factors, forest fire is deliberate in the area for the collection of beedi (tendu) leaf and other NTFPs, like grass.

\section{Materials and methods}

The field trips to the study area were designed to cover the four seasons for three years (2010-2012). Each field visit lasted 4-5 days. The ethnobotanical data were collected through Participatory Rural Appraisal (PRA), which deals the interaction with the indigenous people and direct surveillance in the field (Chambers, 1994). A number of semi-structured interviews from the tribes Yerukalas and Lambadis were conducted in groups or with individuals who are usually above 40 years, with heads of families, house-wives, mid-wives, cattle and/or sheep owners and local vaidyas. The use of plants by the people of 31 villages of the sanctuary was documented (Saidulu, 2014). The information sought was about the uses of plants, e.g. hut construction, agriculture, food, medicine, ornaments, textiles, rituals etc. A total of 59 key-informants have been interviewed as follows: male 48 $(81.36 \%)$ and female 11 (18.64\%), of 30-70 years in five age groups such as 30-39, 40-49, 50-59, 60-69, 70-79 and the percentage (\%) of informants is $8.47(5), 27.12(16), 30.51$ (18), 32.2 (19), 1.7 (01), respectively. The plant specimens with flower, fruit, tuber, rhizome etc. were collected to identify the species and to authenticate the ethnic claims. The voucher specimens of plants of use were collected, processed, labeled and incorporated into Kakatiya University Herbarium (KUW), Warangal (Saidulu, 2014).

Special attention was paid to solicit the field data relating to the habitat, habit, features of flower and fruit, economic/medicinal parts etc. of the plants used by the tribes. Information was documented for wild and cultivated species used by the two tribes in and around the sanctuary for nutrition, day-to-day living, human and ethnoveterinary medicine.

\section{Prior Informed Content (PIC)}

In compliance with CBD (Convention on Biological Diversity), PIC was consisted after relevant discussions by Kakatiya University Authority with the local tribal communities in Northern Telangana about the nature of use of their traditional knowledge. It is an understanding that any profit derived out of the research pursued after their medicinal plant knowledge shall belong to them (Suthari et al., 2014a, b).

\section{Results and discussions}

\section{Ethnobotany}

The present study documented the uses of plants by the local ethnic tribes, the Yerukalas and Lambadis, who reside in 31 villages in and around the Pocharam wildlife sanctuary. Table 1 provides the scientific name followed by family, growth form, occurrence, plant part/s and the traditional use of each and every taxon documented. It was noted that Pocharam wildlife sanctuary was poor in regard to the ethnic diversity, forest 
166

Table 1. Documentation of ethnobotanical plants from Pocharam wildlife sanctuary

\begin{tabular}{|c|c|c|c|c|c|c|}
\hline & Scientific Name & Family & Growth form & Occurrence & $\begin{array}{l}\text { Plant part } / \mathrm{s} \\
\text { used }\end{array}$ & Traditional use \\
\hline & \multicolumn{6}{|l|}{ Magnoliopsida } \\
\hline 1 & Abrusprecatorius & Papilionaceae & Climber & Wild & Seed & Arrow poison, Contraceptive, Scorpion sting \\
\hline 2 & Abutilon indicum & Malvaceae & Shrub & Wild & Root/Leaf & Dysentery/Toothache \\
\hline 3 & Acacia chundra & Mimosaceae & Tree & Wild & Bark/Wood & Evil spirits/Agricultural implements \\
\hline 4 & Acacia farnesiana & Mimosaceae & Tree & Wild & Fruit/Gum & Dog bite/Toothache \\
\hline 5 & Acacia leucophloea & Mimosaceae & Tree & Wild & Bark/Wood & Liquor/Agricultural implements \\
\hline 6 & Acacia nilotica & Mimosaceae & Tree & Planted & Wood/Bark & Commerce, Agricultural implements/ Burns \\
\hline 7 & Acacia torta & Mimosaceae & Shrub & Wild & Root/Bark & Fever/Stupefying fish \\
\hline 8 & Acalypha indica & Euphorbiaceae & Herb & Wild & Root/Leaf & Antiemetic/Skin disease \\
\hline 9 & Achyranthes aspera & Amaranthaceae & Herb & Wild & Leaf & Toothache, Scorpion sting \\
\hline 10 & Aegle marmelos & Rutaceae & Tree & Wild & Fruit/Bark & Cooling effect, Diarrhoea/Dysentery \\
\hline 11 & Aerva lanata & Amaranthaceae & Herb & Wild & Whole plant & Kidney stones, Leafy vegetable \\
\hline 12 & Ailanthus excelsa & Simaroubaceae & Tree & Natur & Bark & Piles, Rheumatism, Skin disease \\
\hline 13 & Alangium salvifolium & Alangiaceae & Tree & Wild & Fruit/Root bark/Wood & Anorexia/Purgative/Fuel \\
\hline 14 & Albizia amara & Mimosaceae & Tree & Wild & Gum & Anti-ulcer \\
\hline 15 & Albizia lebbeck & Mimosaceae & Tree & Planted/R_wild & Wood/Seed & Timber/Toothache \\
\hline 16 & Alternanthera sessilis & Amaranthaceae & Herb & Wild & Whole plant/Shoots & Bone fracture/Leafy Vegetable \\
\hline 17 & Amaranthusspinosus & Amaranthaceae & Herb & E/Natur & Root/Leaf & Rheumatic pains/Vegetable \\
\hline 18 & Ampelocissus latifolia & Vitaceae & Climber & Wild & Stem/Fruit/Leaf & Anorexia/Cooling effect/Vegetable \\
\hline 19 & Andrographis paniculata & Acanthaceae & Herb & Wild & Whole plant & Cold, Fever, Dyspepsia \\
\hline 20 & Anisomeles indica & Lamiaceae & Herb & Wild & Root/Leaf & Centipede bite/Dog bite \\
\hline 21 & Anogeissus latifolia & Combretaceae & Tree & Wild & Wood/Gum & Agricultural implements/Commerce \\
\hline 22 & Aristolochia indica & Aristolochiaceae & Climber & Wild & Root & Chest pain, Snake bite \\
\hline 23 & Atylosia scarabaeoides & Papilionaceae & Herb & Wild & Seed/Whole plant & Vegetable/Fodder \\
\hline 24 & Azadirachta indica & Meliaceae & Tree & Planted/R_wild & Leaf/Branchlet/Wood & Chicken pox/Tooth brush/Agricultural implements \\
\hline 25 & Bacopa monnieri & Scrophulariaceae & Herb & Wild & Whole plant & Cooling effect, Tonic \\
\hline 26 & Balanites roxburghii & Balanitaceae & Tree & Wild & Fruit & Fish poison, Whooping cough \\
\hline 27 & Bambusa arundinacea & Poaceae & Tree & Wild & Culms/Sprouts & Baskets/Food \\
\hline 28 & Barringtonia acutangula & Barringtoniaceae & Tree & Wild & Wood/Leaf/Bark & Boat building/Diarrhoea/Fish poison \\
\hline 29 & Baubinia racemosa & Caesalpiniaceae & Tree & Wild & Leaf/Bark & Beedi wrappers/Cordage \\
\hline 30 & Boerhavia diffusa & Nyctaginaceae & Herb & Wild & Whole plant & Jaundice \\
\hline 31 & Bombax ceiba & Malvaceae & Tree & Wild & Bark/Wood & Anthrax/Utensils \\
\hline 32 & Boswellia serrata & Burseraceae & Tree & Wild & Resin & Commerce, Dog bite, Eruptions \\
\hline 33 & Buchanania axillaris & Anacardiaceae & Tree & Wild & Gum/Kernels & Wounds/Food \\
\hline 34 & Buchanania cochinchinensis & Anacardiaceae & Tree & Wild & Gum/Leaf & Chest pain/Blister, Boils \\
\hline 35 & Butea monosperma & Papilionaceae & Liana & Wild & Flower & Dye, Adoration \\
\hline 36 & Calotropis gigantea & Apocynaceae & Shrub & E/Natur & Latex & Dental care, Migrane, Ear-ache \\
\hline 37 & Canavalia virosa & Papilionaceae & Climber & Wild & Pod/Branch & Food/Galactogogue \\
\hline 38 & Canthium coromandelicum & Rubiaceae & Shrub & Wild & Root bark/Fruit & Dysentery/Food \\
\hline 39 & Capparis zeylanica & Capparaceae & Climber & Wild & Leaf/Root & Boils/Evil spirits \\
\hline 40 & Caralluma adscendensvar. attenuata & Apocynaceae & Herb & Wild & Pulp/Whole plant & Burns/Galactogogue, Fever \\
\hline 41 & Cardiospermum halicacabum & Sapindaceae & Climber & Wild & Leaf/Fruit & Cooling effect/Ringworm \\
\hline 42 & Careya arborea & Barringtoniaceae & Tree & Wild & Bark/Flower & Body swellings/Maternal pain \\
\hline 43 & Carissa spinarum & Apocynaceae & Shrub & Wild & Fruit & Cooling effect \\
\hline 44 & Cassia fistula & Caesalpiniaceae & Tree & Wild & Leaf/Root bark & Dyspepsia/Purgative \\
\hline 45 & Catunaregam spinosa & Rubiaceae & Shrub & Wild & Fruit & Fish poison \\
\hline 46 & Celosia argentea & Amaranthaceae & Herb & E/Natur & Leaf/Inflorescence & Insect bite, Toothache/Worship \\
\hline 47 & Chamaesyce hirta & Euphorbiaceae & Herb & Wild & Whole plant & Boils, Blisters, Cuts, Galactogogue \\
\hline 48 & Chloroxylon swietenia & Flindersiaceae & Tree & Wild & Wood/Leaf & Furniture/Mosquito repellent \\
\hline 49 & Cissus vitiginea & Vitaceae & Shrub & Wild & Stem & Bronchitis, Headache \\
\hline 50 & Cleistanthus collinus & Phyllanthaceae & Tree & Wild & Whole plant/Bark & Fish poison/Wounds \\
\hline 51 & Cleome gynandra & Cleomaceae & Herb & E/Natur & Leaf & Inflammation, Food \\
\hline 52 & Coccinia grandis & Cucurbitaceae & Climber & Wild & Leaf & Diabetes \\
\hline 53 & Cocculus hirsutus & Menispermaceae & Climber & Wild & Leaf & Cooling effect, Food \\
\hline 54 & Cochlospermum religiosum & Cochlospermaceae & Tree & Wild & Bark/Gum & Bone fracture/Ulcers \\
\hline 55 & Corchorus trilocularis & Malvaceae & Herb & Wild & Leaf & Fever \\
\hline 56 & Cordia dichotoma & Boraginaceae & Tree & Wild & Fruit & Dyspepsia \\
\hline 57 & Croton bonplandianum & Euphorbiaceae & Herb & E/Natur & Bark/Latex & Contraceptive/Cuts, Wounds \\
\hline 58 & Cyanthillium cinerareum & Asteraceae & Herb & E/Natur & Leaf & Ring worm \\
\hline 59 & Derris scandens & Papilionaceae & Climber & Wild & Leaf/Root & Fish poison/Rheumatism \\
\hline 60 & Desmodium triflorum & Papilionaceae & Herb & Wild & Root/Leaf & Asthma/Diarrhoea \\
\hline 61 & Dicbrostachys cinerea & Mimosaceae & Shrub & Wild & Leaf/Bark & Boils, Blisters, Cuts/Rheumatism \\
\hline 62 & Diospyros chloroxylon & Ebenaceae & Tree & Wild & Fruit/Bark & Food/Snake bite \\
\hline 63 & Diospyros melanoxylon & Ebenaceae & Tree & Wild & Leaf/Fruit/Wood & Beedi making/Food/Rafters \\
\hline 64 & Diplocyclospalmatus & Cucurbitaceae & Climber & Wild & Fruit & Fever \\
\hline 65 & Dregea volubilis & Apocynaceae & Climber & Wild & Root/Leaf & Rheumatism/Veterinary \\
\hline 66 & Eclipta prostrata & Asteraceae & Herb & Wild & Leaf & Food, Jaundice \\
\hline 67 & Enicostema axillare & Gentianaceae & Herb & Wild & Whole plant & Chronic fever, Diabetes \\
\hline 68 & Euphorbia nivulia & Euphorbiaceae & Tree & Cult & Latex & Rheumatism \\
\hline 69 & Evolvulus alsinoides & Convolvulaceae & Herb & Wild & Whole plant & Aphrodisiac, Mental disorders \\
\hline 70 & Ficus benghalensis & Moraceae & Tree & Wild & Latex & Rheumatism \\
\hline 71 & Ficus hispida & Moraceae & Small tree & Wild & Leaf/Bark & Boils/Galactogogue \\
\hline 72 & Ficus racemosa & Moraceae & Tree & Wild & Fruit/Stem bark & Food/Liquor \\
\hline 73 & Firmiana simplex & Malvaceae & Tree & Wild & Gum/Seed & Commercial, Cooling, Dysentery/Food \\
\hline 74 & Flacourtia indica & Flacourtiaceae & Tree & Wild & Bark/Fruit & Boils, Blisters, Cuts/Food \\
\hline 75 & Gardenia gummifera & Rubiaceae & Tree & Wild & Gum-resin & Snake bite \\
\hline 76 & Gardenia resinifera & Rubiaceae & Tree & Wild & Gum-resin & Fever, Whooping cough \\
\hline 77 & Getonia floribunda & Combretaceae & Climber & Wild & Leaf & Purgative \\
\hline
\end{tabular}




\begin{tabular}{|c|c|c|c|c|c|c|}
\hline 78 & Grewia birsuta & Malvaceae & Shrub & Wild & Root & Boils, Blisters, Cuts \\
\hline 79 & Grewa tiliifolia & Malvaceae & Shrub & Wild & Stem/Fruit/Leaf & Cordage/Dyspepsia/Fodder \\
\hline 80 & Gymnema sylvestre & Apocynaceae & Climber & Wild & Leaf & Diabetes, Opacity of cornea \\
\hline 81 & Hardwickia binata & Caesalpiniaceae & Tree & Wild & Bark/Gum & Cordage/Gonorrhoea \\
\hline 82 & Helicteres isora & Malvaceae & Shrub & Wild & Bark & Cordage \\
\hline 83 & Hemidesmus indicus var. indicus & Apocynaceae & Climber & Wild & Root & Blood purifier \\
\hline 84 & Hemidesmusindiaus var.pubescens & Apocynaceae & Climber & Wild & Root & Galactogogue \\
\hline 85 & Hibiscus sabdariffa & Malvaceae & Herb & Cult & Bark/Leaf & Fibre/Food \\
\hline 86 & Holarrbena pubescens & Apocynaceae & Tree & Wild & Leaf/Bark & Dysentery/Headache \\
\hline 87 & Holoptelea integrifolia & Ulmaceae & Tree & Wild & Wood/Leaf & Fuel/Skin disease \\
\hline 88 & Hybanthus enneaspermus & Violaceae & Herb & Wild & Whole plant/Leaf & Aphrodisiac/Purgative \\
\hline 89 & Hygrophila auriculata & Acanthaceae & Herb & Wild & Root/Leaf & Gonorrhoea/Oedema \\
\hline 90 & Hyptis suaveolens & Lamiaceae & Herb & E/Natur & Whole plant/Dried shoots & Mosquito repellent/Fencing thatching \\
\hline 91 & Ipomoea aquatica & Convolvulaceae & Climber & Wild & Whole plant/Leaf & Cooling effect/Food \\
\hline 92 & Ipomoea carnea ssp. fistulosa & Convolvulaceae & Climber & E/Natur & Whole plant & Fence \\
\hline 93 & Lannea coromandelica & Anacardiaceae & Tree & Wild & Stem bark/Wood & Bone fracture, Foot cracks/Agricultural implements \\
\hline 94 & Lepidagathis cristata & Acanthaceae & Herb & Wild & Root/Whole plant & Body pains/Evil spirits \\
\hline 95 & Limonia acidissima & Rutaceae & Tree & Wild & Fruit & Dysentery, Food, Indigestion, Renal problems \\
\hline 96 & Litsea glutinosa & Lauraceae & Tree & Wild & Bark/Leaf & Bone fracture/Horn cancer \\
\hline 97 & Madhuca longifolia var. Latifolia & Sapotaceae & Tree & Wild & Flower/Seed & Galactogogue, Liquor/Food \\
\hline 98 & Mangifera indica & Anacardiaceae & Tree & Wild & Bark/Fruit & Boils, Blisters, Wounds/Food \\
\hline 99 & Manilkara hexandra & Sapotaceae & Tree & Wild & Fruit/Branch & Cooling effect/Decoration \\
\hline 100 & Maytenus emarginata & Celastraceae & Shrub & Wild & Leaf & Oedema \\
\hline 101 & Miliusa tomentosa & Annonaceae & Tree & Wild & Wood & Agricultural implements \\
\hline 102 & Morinda pubescens & Rubiaceae & Tree & Wild & Wood/Root bark & Agricultural implements/Stomach ache \\
\hline 103 & Mucuna pruriens var. hirsuta & Papilionaceae & Climber & Wild & Root & Centipede bite, Filaria \\
\hline 104 & Mukia maderaspatana & Cucurbitaceae & Climber & Wild & Fruit/Leaf & Blood purification/Dandruff \\
\hline 105 & Nelumbo nucifera & Nymphaeaceae & Herb & Wild & Leaf & Epistaxis \\
\hline 106 & Nymphaea nouchali & Nymphaeaceae & Herb & Wild & Flower & Adoration, Eye infection \\
\hline 107 & Ocimum americanum & Lamiaceae & Herb & E/Cult/Natur & Leaf & Mosquito repellent \\
\hline 108 & Ocimum tenuiflorum & Lamiaceae & Herb & E/Cult & Leaf & Cold, Cough, Malaria \\
\hline 109 & Olax scandens & Olacaceae & Shrub & Wild & Leaf/Fruit & Diarrhoea/Food \\
\hline 110 & Pavonia zeylanica & Malvaceae & Shrub & Wild & Leaf & Diuretic \\
\hline 111 & Pergularia daemia & Apocynaceae & Climber & Wild & Leaf/Latex & Leprosy/Rheumatism \\
\hline 112 & Phyla nodiflora & Verbenaceae & Herb & Wild & Leaf/Whole plant & Kidney stones/Toothache \\
\hline 113 & Phyllanthus amarus & Phyllanthaceae & Herb & E/Natur & Whole plant & Diabetes, Jaundice \\
\hline 114 & Phyllanthus emblica & Phyllanthaceae & Tree & Wild & Fruit & Food, Dandruff \\
\hline 115 & Phyllanthus reticulatus & Phyllanthaceae & Shrub & Wild & Leaf/Shoots & Fodder/Making baskets \\
\hline 116 & Physalis angulata & Solanaceae & Herb & E/Natur & Fruit & Intestinal disorders, Purgative \\
\hline 117 & Pithecellobium dulce & Mimosaceae & Tree & Planted & Wood/Arils & Agricultural implements/Blood purification \\
\hline 118 & Polycarpea corymbosa & Caryophyllaceae & Herb & Wild & Leaf & Inflammation, Jaundice \\
\hline 119 & Pongamia pinnata & Papilionaceae & Tree & Wild/Planted & Seed/Shoots & Commerce/Tooth brush \\
\hline 120 & Portulaca oleracea & Portulacaceae & Herb & Wild & Shoot & Leafy vegetable \\
\hline 121 & Portulaca quadrifida & Portulacaceae & Herb & Wild & Leaf & Burns, Skin disease \\
\hline 122 & Prosopis cineraria & Mimosaceae & Tree & Wild & Shoots, Leaf/Wood & Ceremonial/Fuel \\
\hline 123 & Prosopis juliflora & Mimosaceae & Tree & $\mathrm{E} /$ Natur & Fruit/Wood & Fodder/Fuel wood \\
\hline 124 & Pterocarpus marsupium & Papilionaceae & Tree & Wild & Gum/Wood/Bark & Adhesive/Agricultural implements/Cordage \\
\hline 125 & Rauvolfia serpentina & Apocynaceae & Shrub & Wild & Root & Centipede bite, Snake bite \\
\hline 126 & Ricinus communis & Euphorbiaceae & Shrub & E/Cult/R_wild & Seed/Leaf & Constipation/Sun stroke \\
\hline 127 & Rivea hypocrateriformis & Convolvulaceae & Climber & Wild & Root/Leaf & Dog bite/Piles \\
\hline 128 & Sapindus emarginatus & Sapindaceae & Tree & Wild & Leaf/Fruit & Cooling effect/Dandruff \\
\hline 129 & Schleichera oleosa & Sapindaceae & Tree & Wild & Bark/Leaf & Chest pain, Sores/Ulcers \\
\hline 130 & Semecarpus anacardium & Anacardiaceae & Tree & Wild & Hypocarp/Seed & Food/Magico religious beliefs \\
\hline 131 & Senna auriculata & Caesalpiniaceae & Shrub & Wild & Seed/Flower & Adoration/Diabetes \\
\hline 132 & Sida acuta & Malvaceae & Herb & Wild & Whole plant & Broom \\
\hline 133 & Sida cordata & Malvaceae & Herb & Wild & Whole plant/Leaf & Dysentery/Scorpion sting \\
\hline 134 & Solanum americanum & Solanaceae & Shrub & E/Natur & Leaf/Fruit & Food/Skin disease \\
\hline 135 & Solanum virginianum & Solanaceae & Climber & E/Natur & Root/Stem/Fruit & Leprosy/Cough/Worm killing \\
\hline 136 & Solanum torvum & Solanaceae & Shrub & R_Wild & Fruit & Vegetable \\
\hline 137 & Solena amplexicaulis & Cucurbitaceae & Climber & Wild & Whole plant & Diabetes \\
\hline 138 & Soymida febrifuga & Meliaceae & Tree & Wild & Bark/Wood & Agricultural implements, Fuel/Diarrhoea \\
\hline 139 & Sphaeranthus indicus & Asteraceae & Herb & Wild & Whole plant & Lice killing, Stomach ache \\
\hline 140 & Strychnos nux-vomica & Loganiaceae & Tree & Wild & Bark/Seed & Magico religious beliefs, Poison/Snake bite \\
\hline 141 & Strychnos potatorum & Loganiaceae & Tree & Wild & Seed & Detergent, Water purification \\
\hline 142 & Syzygium cumini & Myrtaceae & Tree & Wild & Branch/Fruit pulp/Wood & Adoration/Eye sight/Making carts \\
\hline 143 & Tectona grandis & Lamiaceae & Tree & Wild & Inflorescence/Wood & Adoration/Commerce \\
\hline 144 & Tephrosia purpurea & Papilionaceae & Herb & Wild & Root & Fever \\
\hline 145 & Terminalia arjuna & Combretaceae & Tree & Wild & $\begin{array}{l}\text { Wood/Stem bark/Whole } \\
\text { plant }\end{array}$ & $\begin{array}{l}\text { Agricultural implements/Boils, blisters/Magico } \\
\text { religious beliefs }\end{array}$ \\
\hline 146 & Terminalia bellirica & Combretaceae & Tree & Wild & Gum, Fruit/Whole plant & Commerce/Evil spirits \\
\hline 147 & Tinospora cordifolia & Menispermaceae & Climber & Wild & Root/Stem & Cough, Galactogogue/Fever \\
\hline 148 & Tribulus lanuginosus & Zygophyllaceae & Climber & Wild & Root/Fruit & Cooling, Demulcent/Gonorrhea \\
\hline 149 & Trichosanthes cucumerina & Cucurbitaceae & Climber & Wild & Fruit & Skin disease \\
\hline 150 & Tridax procumbens & Asteraceae & Herb & E/Natur & Leaf/Whole plant & Boils, Blisters, Cuts/Wounds \\
\hline 151 & Tylophora indica & Apocynaceae & Climber & Wild & Leaf/Root & Asthma/Cough, Jaundice \\
\hline 152 & Ventilago denticulata & Rhamnaceae & Climber & Wild & Leaf/Stem bark & Debility/Stomach ache \\
\hline 153 & Vitex negundo & Verbenaceae & Tree & Planted & Leaf & Arthritis, Headache, Post-partum problems \\
\hline 154 & Wrightia tinctoria & Apocynaceae & Tree & Wild & Bark/Wood & Boils, Blisters, Wounds/Toys, decorative \\
\hline 155 & Xanthium indicum & Asteraceae & Herb & E/Natur & Root/Whole plant & Evil spirits/Sores \\
\hline 156 & Xylia xylocarpa & Mimosaceae & Tree & Wild & Wood/Leaf & Agricultural implements/Purgative \\
\hline 157 & Ziziphus oenopolia & Rhamnaceae & Climber & Wild & Fruit/Stem bark & Edible/Mouth wash \\
\hline 158 & Ziziphus xylopyrus & Rhamnaceae & Tree & Wild & Leaf/Fruit & Fodder/Wounds \\
\hline
\end{tabular}




\begin{tabular}{|c|c|c|c|c|c|c|}
\hline & Liliopsida & & & & & \\
\hline 159 & Aloevera & Xanthorrhoeaceae & Herb & Planted & Leaf & Burning urine, Menstrual troubles, Piles \\
\hline 160 & Asparagus racemosus & Asparagaceae & Herb & Wild & Tuber & Dyspepsia, Ulcers \\
\hline 161 & Borassus flabellifer & Arecaceae & Tree & Natur & Sap/Leaf/Petiole & Toddy/Mats, Baskets/Fibre \\
\hline 162 & Chlorophytum tuberosum & Amaryllidaceae & Herb & Wild & Tuber & Dysentery, Hydrocele \\
\hline 163 & Crinum asiaticum & Amaryllidaceae & Herb & Wild & Tuber & Snake bite, Wounds \\
\hline 164 & Curculigo orchioides & Hypoxidaceae & Herb & Wild & Tuber & Aphrodisiac, Inflammation, Piles \\
\hline 165 & Curcuma pseudomontana & Zingiberaceae & Herb & Wild & Tuber/Leaf & Jaundice/Meal plates, Wounds \\
\hline 166 & Cynodon dactylon & Poaceae & Herb & Wild & Whole plant & Cold, Cough, Fodder \\
\hline 167 & Cyperus rotundus & Cyperaceae & Herb & Wild & Tuber & Ephemeral fever \\
\hline 168 & Dendrocalamus strictus & Poaceae & Tree & Wild & Whole plant/Grains & Construction/Food \\
\hline 169 & Dioscorea bulbifera & Dioscoreaceae & Climber & Wild & Tuber & Food \\
\hline 170 & Dioscorea oppositifolia & Dioscoreaceae & Climber & Wild & Tuber & Food \\
\hline 171 & Gloriosa superba & Colchicaceae & Climber & Wild & Leaf/Tuber & Lice eradication/Rheumatism \\
\hline 172 & Phoenix sylvestris & Arecaceae & Tree & Wild & Fruit/Leaf/Sap & Cooling effect/Making mats, Thatching/ Toddy \\
\hline 173 & Vanda tessellata & Orchidaceae & Herb & Wild & Whole plant & Ephemeral fever, Evil spirits \\
\hline
\end{tabular}

Table 2. Five top-rank angiosperm families used in Pocharam wildlife sanctuary

\begin{tabular}{ccc}
\hline $\begin{array}{c}\text { No. } \\
\text { crt. }\end{array}$ & $\begin{array}{c}\text { No. of species of } \\
\text { traditional use }\end{array}$ & Family/Families \\
\hline 1 & 26 & $\begin{array}{c}\text { Leguminosae/Fabaceae }(10 \\
\text { Papilionaceae }+ \text { 04 Caesalpiniaceae }+ \\
12 \text { Mimosaceae) }\end{array}$ \\
2 & 12 & $\begin{array}{c}\text { Apocynaceae* (incl. 08 of } \\
\text { Asclepiadaceae) }\end{array}$ \\
3 & 11 & $\begin{array}{c}\text { Malvaceae* (incl. 01 Bambacaceae+ } 03 \\
\text { Tiliaceae }+ \text { 01 Sterculiaceae) }\end{array}$ \\
4 & 05 & $\begin{array}{c}\text { Amaranthaceae, Anacardiaceae, } \\
\text { Asteraceae, Cucurbitaceae, } \\
\text { Euphorbiaceae and Lamiaceae } \\
\text { Phyllanthaceae* }\end{array}$ \\
5 & 04 &
\end{tabular}

diversity, or its stakeholder botanical knowledge (Saidulu, 2014) in contrast to the Eturnagaram wildlife sanctuary in the neighbouring Warangal district, wherein more than 200 species are used only for medicinal use by Koya community alone (Suthari et al.,2014b).

\section{Floristic/Taxonomic account}

The survey of the sanctuary area resulted in data record of 173 angiosperm (Magnoliophyta) species that are in use by the two ethnic communities. These species belong to 148 genera and 68 families as per the traditional system of classification of Takhtajan (1980) (Table 1). Of these, 57 families, 133 genera and 158 species are of Dicotyledonae (Magnoliopsida) and 11 families, 15 genera and 15 species are of Monocotyledonae (Liliopsida). The ratio of Dicots and Monocots of ethnic use is 10.5:1 for species and 5.2:1 for families. Conversely, as realized and recorded in other studies (Suthari et al., 2014b), the Dicots contribute more than Monocots to the medicinal and ethnobotanical use. This might be due to the species strength in the region.

When one looks at the use-contribution from the angiosperm families, Leguminosae (incl. 12 of Mimosaceae, 10 of Papilionaceae and 04 of Caesalpiniaceae) tops the list, followed by Apocynaceae (incl. Asclepiadaceae as per APG III, 2009). The third position is occupied by Malvaceae (s.l.), followed by Amaranthaceae, Anacardiaceae, Asteraceae, Cucurbitaceae, Euphorbiaceae and Lamiaceae with the same rank, and then by Phyllanthaceae (Table 2). However, Reddy et al. (2010) found Asclepiadaceae ( 5 species), Amaranthaceae and Caesalpiniaceae (4 species each) as the dominant families contributing to ethnomedicine by ethnic tribes in Medak district.

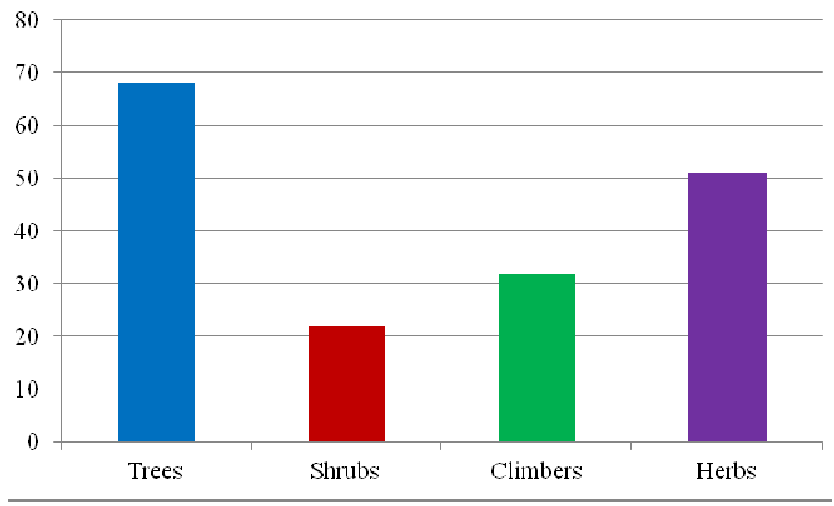

Fig. 2. Plant-use pattern in regard to growth forms

\section{Plant growth forms}

Plants are usually classified as trees, shrubs, climbers and herbs, as growth forms. When the plant use-data were analyzed, trees contributed with 68 uses, followed by herbs (51), climbers (32) and shrubs (22). Perhaps this was a reflection of the floristic composition and the prevailing Phanero-therophytic climate (Raju et al., 2014), where the trees and annuals co-dominate in a disturbed tropical forest habitat. It can also be inferred that the ethnic people use the locally abundant vegetation (e.g. plant cover). Furthermore, in a habitat where trees are displaced, the preponderance of species increased from shrubs to herbs via climbers (Fig. 2). While the annuals were seasonal, the other growth forms were available throughout the year for use.

\section{Ethnoagriculture}

The people of Pocharam wildlife sanctuary do not indulge in the usual practice of Podu (slash and burn) cultivation, since a large part of the land area is already under modern agriculture. However, they use the local minor wood for making agricultural implements, construction of huts, putting fences to homes and fields etc. Besides, it was noted that the local people no longer cultivate the traditional or indigenous varieties of grains.

\section{Indigenous vs. Exotics}

Out of the 173 plant taxa that were noted as being utilized by the ethnic people in the sanctuary, the greatest number (154; 89.1\%) were indigenous and wild. The introduced species were the crops under cultivation and those planted (Table 1). The ratio of indigenous to exotics species of use was 12:1. As many as 154 indigenous taxa and 13 exotics (7.51\%) are used besides the four species (Acacia nilotica, Aloe vera, Pongamia pinnata and 


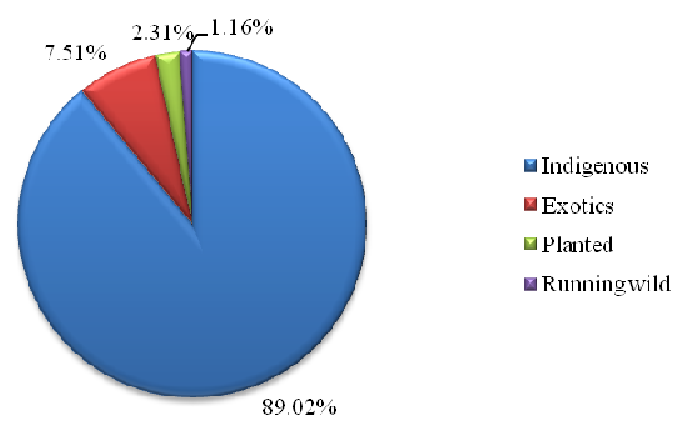

Fig. 3. Indigenous vs. exotic ethnobotanical plants in the sanctuary

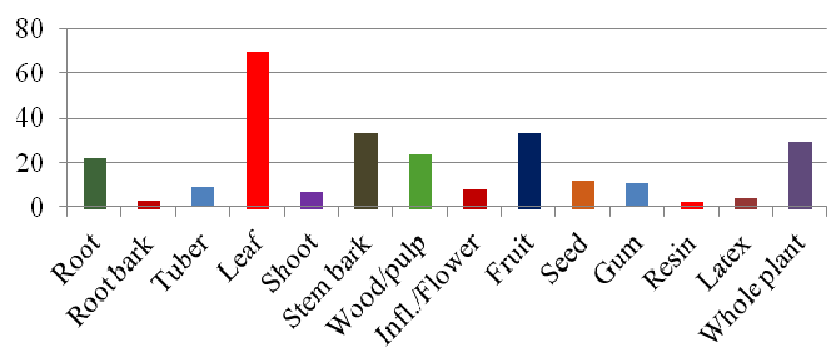

Fig. 4. Plant part-wise for ethnic use

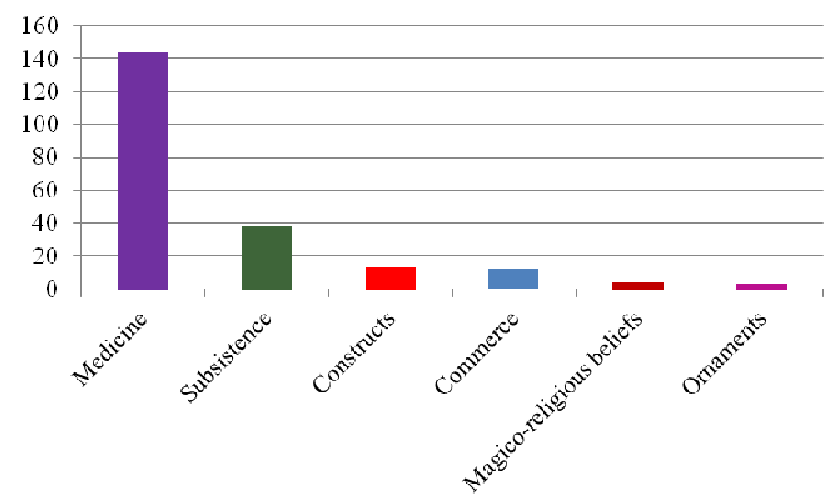

Fig. 5. Ethnic-use pattern and proportions in Pocharam wildlife sanctuary

Vitex negundo) that are planted (2.31\%) and two $(1.16 \%)$ species (Albizia lebbeck and Azadirachta indica) that are running wild and naturalized. Obviously, the ethnic people were basically using the indigenous wild species (Fig. 3).

\section{Plant part-wise use}

The following is the plant part-wise analysis of ethnobotanical uses (Fig. 4): (a) Roots: These are used as whole (22), bark (03), or as tubers (09), numbering a total of 34 species; (b) Shoots: Of the above ground parts, leaves are used in 69 cases, fruits and stem bark (33 of each), wood/pulp (24), seeds (12), inflorescence/flower (08) and shoots (07); (c) Whole plant: There are 29 species used for medicinal and/or consumptive purposes; and (d) Un-organized (latex, gum and resin): Gum is obtain from 11 taxa, whereas gum-resin from only 2 . The latex of 4 species is used (Table 1).

\section{Ethnic-usepattern}

Although the local people use plants for various purposes, they largely serve medicinal scopes and for subsistence. The analysis of the baseline data indicated the following facts: (a)
Medicine: There were 140 species used for human medicine, either as single drug or in combinations. There were four species of use as ethnoveterinary medicine; (b) Subsistence: Of the 173 flowering plant taxa, 27 were used as food and/or vegetables, and 11 to catch (stupefy) fish; (c) Constructs: Plants are used to find additional value by their creative skills, e.g. make boats, carts, cots, agricultural implements and other household things. There were 13 tree species utilized for making agricultural implements and five for house/hut construction (Table 1); (d) Commerce: For the economic subsistence, during the off-season or nonagricultural season, people gather plant parts (leaves, flowers, fruits, seeds, bark, etc.) or extract through manipulation gums, resins and latex, in order to sell them to Girijan Cooperative Corporation Limited (GCC) or in the local candies. There were 12 such species of this category (Table 1). (e) Magic/ritualreligious beliefs: There are deep-rooted beliefs in the ethnic communities. Four species are used to get cured of a disease and six species to banish evil spirits; and (f) Ornaments: There were only three species used as ornaments (Table 1). However, most of the plant species used by Yerukalas and Lambadis were of medicinal (83.24\%) and subsistence (21.96\%) value (Fig. 5).

\section{Conclusions}

The present study has yielded the baseline ethnobotanical data about a wildlife sanctuary in Northern Telangana after its six decades of standing, which is not in its prime and best care. Besides, the local forest-loving Gonds are displaced or wanted. The study clearly revealed that the extant tropical dry deciduous forest is not intact for the terrain and phytoclimate under state protection. It is to be reinstated that Pocharam wildlife sanctuary is very poor in regard to the ethnic diversity, forest diversity, or its stakeholder botanical knowledge. Gathering the forest produce for commerce is more so over their self-use, e.g. for household purposes, community beliefs and ornaments. However, it was interesting to note that the NTFPs are still providing a succor to the local people. It is recommended that cummunities's participation is to be called for the protection of the existing vegetation in the sanctuary, and watersheds are to be developed wherever feasible, while allowing the stakeholders to gather some of the forest produce (NTFPs), to encourage and educate them for mutual benefits. Thereby, ecological sustainability (functional) of the sanctuary is promoted.

\section{Acknowledgements}

The authors thank Mr K. Sarveshar, Forest Range Officer, Pocharam, Telangana State Forest Department, for permission to stay in the forest guest house and enthusiastic support. The authors are obliged to all the informants of the study area for sharing their traditional botanical knowledge. The authors thank the Head, Department of Botany, Kakatiya University, Warangal, for facilities.

\section{References}

Anonymous (2000). Notification of the Wildlife Sanctuary (Fascimile)Pocharam Wildlife Sanctuary. The Andhra Pradesh Gazetter, PartI - Extraordinary, published by Authority, Hyderabad.

APG III [The Angiosperm Phylogeny Group] (2009). An update of the Angiosperm phylogeny group classification for the orders and families of flowering plants: APG III. Botanical J Linn Soc 161:105-121. 
170

Chambers R (1994). Participatory rural appraisal (PRA): Analysis of experience. World Develop 22(9):1253-1268.

Hemadri K (1990). Contribution to the medicinal flora of Karimnagar and Warangal districts of Andhra Pradesh. Indian Med 2:16-28.

Jain SK (1999). Dictionary of Ethnoveterinary Plants of India. Deep Publications, New Delhi.

Kapoor SL, Kapoor LD (1980). Medicinal plant wealth of the Karimnagar district of Andhra Pradesh. Bull Medico-Ethnobot Res 1:120-144.

Khan MS (1953). Forest Flora of Hyderabad State. Govt Press, Hyderabad.

Kumar DCT, Pullaiah T (1998). Ethnomedicinal uses of some plants of Mahabubnagar district, Andhra Pradesh. J Econ Tax Bot 23:341345.

Murthy EN, Reddy CS, Reddy KN, Raju VS (2007). Plants used in ethnoveterinary practices by Koyas of Pakhal Wildlife Sanctuary, Andhra Pradesh, India. Ethnobotanical Leaflets 11:1-5 (http://www.siu.edu/ ebl/leaflets/ghats.html).

Naqvi AH (2001). Flora of Karimnagar district, Andhra Pradesh, India. Kakatiya Univ, Warangal, $\mathrm{PhD}$ Thesis.

Omkar K, Suthari S, Alluri S, Ragan A, Raju VS (2011). Diversity of NTFPs and their utilization in Adilabad district of Andhra Pradesh, India.J Plant Studies 1(1):33-46.

Padmarao P, Reddy PR (1999). A note on folk treatment of bone fractures in Ranga Reddy district, Andhra Pradesh. Ethnobot 11:107-108.

Pullaiah T, Kumar DCT (1996). Herbal plants in Mannanur forest of Mahabubnagar district, Andhra Pradesh. J Econ Tax Bot (Additional Ser) 12:218-220.

Raju VS, Krishna PG, Suthari S (2014). Environmental assessment of climate of a habitat through floristic life-form spectra, a case study of Warangal North Forest Division, Telangana, India. J Natur Sci 2(1):77-93.

Raju VS, Reddy KN (2005). Ethnomedicine for dysentery and diarrhea from Khammam district of Andhra Pradesh, India. Indian J Trad Knowl 4(4):443-447.

Ramarao N (1988). The ethnobotany of Eastern Ghats in Andhra Pradesh, India. Bharathiar Univ, Coimbatore, PhD Thesis.

Ravishankar T (1990). Ethnobotanical studies in Adilabad and Karimnagar districts of Andhra Pradesh, India. Bharathiar Univ, Coimbatore, PhD Thesis.

Ravishankar T, Henry AN (1992). Ethnobotany of Adilabad district, Andhra Pradesh, India. Ethnobot 4:45-52.

Reddy CS (2002). Floristics Studies in Warangal district, Andhra Pradesh, India. Kakatiya Univ, Warangal, PhD Thesis.
Reddy CS, Raju VS (2000). Folklore biomedicine for common veterinary diseases in Nalgonda district, Andhra Pradesh. Ethnobot 12:113-117.

Reddy CS, Nagesh K, Reddy KN, Raju VS (2002). Plants used in ethnoveterinary practices by Gonds of Karimnagar district, Andhra Pradesh, India. JEcon Tax Bot 27(3):631-634.

Reddy CS, Gopalkrishna P, Raju VS (2007). Phytotherapy at rural communities: a case study from the Gonds of Warangal district, Andhra Pradesh, India. Res J Bot 3(2):97-102.

Reddy KN (2002). Ethnobotany in Khammam district of Andhra Pradesh, India. Kakatiya Univ, Warangal, PhD Thesis.

Reddy KN, Bhanja MR, Raju VS (1998). Plants used in ethnoveterinary practices in Warangal district, Andhra Pradesh, India. Ethnobot 10:75-84.

Reddy KN, Raju VS (2002). Ethnobotanical observations on Konda Reddis of Mothugudem in Khammam district, Andhra Pradesh. Abstract published in National seminar on Conservation of Eastern Ghats 24-26 ${ }^{\text {th }}$ March, Tirupati.

Reddy KN, Reddy CS, Jadhav SN (2001). Heterostemma deccanense (Talb.) Swarup \& Mangaly (Asclepiadaceae): an endangered and endemic taxon in Andhra Pradesh. Indian Forester 127:1403-1404.

Reddy KN, Trimurthulu G, Reddy CS (2010). Medicinal plants used by ethnic people of Medak district, Andhra Pradesh. Indian J Trad Knowl 9(1):184-190.

Saidulu P (2014). Ethnobotany of Pocharam Wildlife Sanctuary, Andhra Pradesh. Kakatiya Univ, Warangal, MPhil Diss.

Sreeramulu N, Suthari S, Omkar K, Raju VS (2013). Ethno-botanicomedicine for common human ailments in Nalgonda and Warangal districts of Andhra Pradesh, India. Ann PI Sci 2(7):220-229.

Suthari S, Sreeramulu N, Omkar K, Raju VS (2014a). The climbing plants of northern Telangana in India and their ethnomedicinal and economic uses. Indian J Pl Sci 3(1):86-100.

Suthari S, Sreeramulu N, Omkar K, Reddy CS, Raju VS (2014b). Intracultural cognizance of medicinal plants of Warangal North Forest Division, Northern Telangana, India. Ethnobot Res Appli 12:211-235.

Swamy NSN (2009). Ethnobotanical knowledge from Adilabad district, Andhra Pradesh, India. Andhra Univ, Visakhapatnam, $\mathrm{PhD}$ Thesis.

Takhtajan A (1980). Outline of the classification of flowering plants (Magnoliophyta). Botanical Rev 46:235-359.

Upadhyay R, Chauhan SVS (2000). Ethnobotanical observations on Koya tribe of Gundala Mandal of Khammam district, Andhra Pradesh. Ethnobot 12:93-99. 